\title{
Kultur und unternehmerisches Handeln. Die Perspektive der „Transkulturalität als Praxis“
}

Die vorliegende Arbeit zeigt, dass ökonomisches Handeln immer auch als kulturelles Handeln gelesen werden kann und dass das Konzept der „Transkulturalität als Praxis“ geeignet erscheint, um die einleitend definierte „kulturelle Dimension“ ökonomischer Handlungspraxis theoriegeleitet zu strukturieren und für die empirische Forschungspraxis erschließbar zu machen. Damit lassen sich wirtschaftsgeographische Fragestellungen um eine wichtige Perspektive erweitern.

„Transkulturalität als Praxis“ basiert auf einem Kulturbegriff, der Kultur als Wissensbestände, symbolische Ordnungen, Deutungsschemata auffasst, über die Menschen notwendigerweise verfügen, um sich Gegenstände oder Handlungen als sinnhaft erschließen zu können. Nur über Kultur ist „Welt“ erfahrbar und damit ist sie Bedingung jeglicher sozialer Praxis: Wenn zwei Menschen aufeinander zugehen und sich gegenseitig mit der rechten Hand anfassen, wird dies von vielen als Ritual der Begrüßung „verstanden“; die Körperbewegung wird als sinnhaft erschlossen. Wie das Beispiel zeigt, müssen solche Deutungsschemata notwendigerweise kollektiv geteilt sein.

Genauso kommt auch die ökonomische Handlungspraxis nicht ohne Rückgriff auf geteilte symbolische Ordnungen aus. Denn in Interaktionssituationen, z.B. einer geschäftlichen Vereinbarung zwischen zwei Unternehmern, lässt sich nur dann ein für die Beteiligten befriedigendes Ergebnis erzielen, wenn bezüglich der Einschätzung dessen, wie z.B. Absprachen besiegelt werden und wie verbindlich sie sind, zwischen den Geschäftspartnern Bedeutungsgleichheit besteht. In diesem Sinne ist jegliches ökonomisches Handeln immer auch kulturelles Handeln. 
Der Umstand, dass Deutungsschemata notwendigerweise kollektiv geteilt sein müssen, erweist sich konzeptionell jedoch als problematisch. Er führt in mancher Arbeit, die sich mit der kulturellen Dimension z.B. ökonomischen Handelns beschäftigt, zu dem Fehlschluss, Kultur als ein geschlossenes System aufzufassen, d.h. als ein Verweisungszusammenhang von Symbolen, der in sich geschlossenen ist. Das beinhaltet häufig, Kultur konzeptionell an abgrenzbare soziale Kollektive zu binden und territorial zu verorten bzw. auf einen territorial verortbaren Ursprung zurückzuführen („türkische Kultur“, „Regionalkultur“).

In der jüngeren kulturtheoretischen Debatte ist von verschiedener Seite darauf aufmerksam gemacht worden, dass eine solche Auffassung von Kultur als geschlossenes System aus einer theoretischen Perspektive nicht haltbar ist. Auch wird dadurch meist die Suche nach dem „Besonderen der Kultur“ zur wichtigsten Forschungsfrage, was essentialistischen Kulturvorstellungen Vorschub leistet und dazu führt, Differenzen „zwischen den Kulturen“ herzustellen und das vermeintliche Anders-Sein von Angehörigen „fremder Kulturen“ zu betonen. Dadurch besteht letztlich die Gefahr, Ausgrenzung und Diskriminierung zu befördern, was die Annahme homogener Kulturen auch gesellschaftspolitisch problematisch werden lässt.

Gleichzeitig muss man aber akzeptieren, dass Essentialisierungen und das Denken in homogenen Kulturen die alltägliche Handlungspraxis maßgeblich prägen. Denn diskursiv vermittelte Deutungsmuster, wie „Welt“ zu verstehen ist, gehen immer einher mit machtvollen Klassifikationsschemata, in die Akteure Handlungen und andere Menschen permanent einordnen. Solche Zuschreibungsprozesse, z.B. entlang der Herkunft, reduzieren Komplexität und stellen dadurch Handlungssicherheit her. Sinnbildung ist auf der Ebene des Handelnden also untrennbar verbunden mit Klassifikation.

Die theoretische Position der Nicht-Existenz homogener Kulturen deckt sich demnach nicht mit der Position handelnder Akteure. Damit besteht die auf den ersten Blick paradoxe Situation, dass es konzeptionell das nicht geben kann, was in der sozialen und diskursiven Praxis laufend hergestellt wird. Genau in diesem Widerspruch ist das Konzept der Transkulturalität angesiedelt, wie es Welsch (1992 und 1999) zu Grunde gelegt hat und wie es in der vorliegenden Arbeit mit „Transkulturalität als Praxis“ für die Untersuchung un- 
ternehmerischen Handelns weiterentwickelt worden ist. „Transkulturalität als Praxis" eröffnet eine Perspektive, welche die Kritik an essentialistischen Kulturkonzeptionen teilt und auf der analytischen Ebene den Blick auf die Grenzen richtet, die Akteure in der Handlungspraxis produzieren und reproduzieren.

Schlüssel dazu ist die Verschiebung der theoretischen Lage kultureller Grenzen von einer interpersonalen auf eine intrapersonale Ebene. Damit wird konzeptionell anerkannt und zugänglich, was sich empirisch in den Interviews für diese Arbeit immer wieder zeigte: Menschen beteiligen sich permanent an der Konstruktion vermeintlich homogener Kulturen und können sich selbst situativ unterschiedlichen dieser imaginären Gemeinschaften zuordnen, ohne dass sie schwerwiegende Identitätskonflikte erleiden würden.

Kultur im Sinne von „Transkulturalität als Praxis“ für empirische Untersuchungen nutzbar machen heißt also, den Blickwinkel zu verlagern. Und zwar weg von vermeintlich gegebenen kulturellen Ausprägungen - denn das hieße, diskursiv konstruierte alltagsweltliche Essentialisierungen als naturhaft Gegebenes anzunehmen und sie in eigenen Untersuchungen zu reproduzieren - und stattdessen hin $\mathrm{zu}$ den Grenzziehungen, die Akteure in ihrem Handeln laufend vornehmen. Mit dem Perspektivenwechsel rückt die Frage in den Vordergrund, an welchen Symbolen diese Grenzen markiert werden. Denn nur sie geben Aufschluss über Zuordnungs- und Ausschließungspraktiken im Sinne von „wer oder was ist außen“ oder „wer oder was gehört wozu" und sind damit Basis für Handlungsverstehen.

Trotz der Verschiebung des Blickwinkels erlaubt die hier zu Grunde gelegte Konzeptualisierung von Kultur, an bestehende Erklärungsmodelle des „Immigrant Business“ anzuknüpfen. Sie sind jedoch dahingehend zu reinterpretieren, dass bei den dominierenden Ansätzen - „strukturelle Rahmenbedingungen“, „Nischenmarktorientierungen“ und „Netzwerkbeziehungen“ - die (Re-)Produktion kultureller Grenzen zu betrachten ist - und zwar auf drei Ebenen, die miteinander zusammenhängen: auf der Ebene diskursiver Felder, auf der Ebene von Institutionen und auf der Ebene konkreter Interaktionssituationen.

So sind Klassifikationsschemata wie jegliche als „Kultur“ definierte Wissensordnungen in Diskursen verankert und hierdurch kollektiv geteilt, verfügbar und wirksam. Sie werden darüber hinaus 
durch Gesetze und Verwaltungsvorschriften institutionalisiert und damit strukturell verfestigt - z.B. durch das Ausländerrecht, welches das Subjekt des „Ausländers“ erst konstituiert und ihm in fast allen öffentlich regulierten Lebensbereichen eine Sonderbehandlung zukommen lässt. Eine solche institutionalisierte Ethnisierung macht sich praktisch beispielsweise darin bemerkbar, dass Menschen ohne entsprechende Aufenthaltstitel der Zugang zu vielen Segmenten des Arbeitsmarktes verwehrt und Selbständigkeit im Regelfall nicht erlaubt wird. Beides kann dazu führen, dass als Handlungsoption für viele nur eine Beschäftigung im informellen Sektor bleibt: entweder durch Annahme einer Schwarzarbeit oder durch Gründung eines Unternehmens über Dritte, womit diverse Abhängigkeitsverhältnisse eingegangen werden müssen.

Wie diskursiv verankerte Klassifikationsschemata entlang national etikettierter Grenzen unternehmerische Handlungsrelevanz entfalten, wird anhand der Konstruktion von „Nischenmärkten“ und „sozialen Ressourcen und Netzwerken“ nachgezeichnet:

- Bei der Konstruktion eines „türkisch“ abgegrenzten Marktes, auf den sich die Marktbearbeitung vieler Unternehmer konzentriert, schlagen sich dominante Diskurse in mehrfacher Hinsicht nieder. Einerseits werden Arbeitsfelder ethnisch konnotiert (Döner schneiden „gehört“ zu Türken), was dazu führt, dass Unternehmensgründungen in spezifischen Bereichen ökonomisch sinnvoller erscheinen als in anderen. Andererseits werden sie in konkreten Interaktionssituationen reproduziert. Wie die Analyse zeigte, müssen dabei die situativ existierenden Machtasymmetrien wie Auftraggeber-Kunde oder Chef-Mitarbeiter beachtet werden, in deren Rahmen sie vermittelt werden. Wenn z.B. der Versicherungsagent Veli in einer Strategiesitzung mit seinen Kollegen gegen seinen Wunsch die Betreuung türkischer Kunden zugewiesen bekommt, weil er türkischer Herkunft ist (und nicht auf die Betreuung von Sachversicherungen, weil er dort die größte Kompetenz besitzt), dann ist dies nichts anderes als die Reproduktion eines dominanten Ethnisierungsdiskurses, der über Machtasymmetrien durchgesetzt und dadurch auch weiter zementiert wird.

- Solche Vergemeinschaftungen entlang der Herkunft sind aber nicht nur Ergebnis von Fremd-, sondern ebenso Resultat von 
Selbstzuschreibungen; wobei beide konzeptionell kaum voneinander getrennt werden können. Auf der Ebene des Subjekts ergeben sich Selbstethnisierungen aus der Dynamik zwischen Annahme und Ablehnung diskursiv konstruierter Identitätspositionen. Sie zeigten sich ebenfalls bei Nischenmarktorientierungen (vgl. insbesondere Mehmet, Fall 4), traten noch klarer aber bei der Konstruktion vermeintlich „türkischer" sozialer Ressourcen hervor. So machte der Blick auf die Situiertheit des unternehmerischen Handelns in sozialen Beziehungen die ökonomische Relevanz der Konstruktion von Grenzen deutlich. Die Herstellung von Zugehörigkeit zu imaginären Gemeinschaften entlang national etikettierter Grenzen dient der Akkumulation sozialen Kapitals, vornehmlich Reziprozität und (erzwingbares) Vertrauen, und der Reduktion von Komplexität und Unsicherheit. Wie gesehen, wirken solche Ressourcen jedoch nur bis zu einem gewissen Grade positiv (z.B. in der Gründungsphase eines Unternehmens), da sie die Handlungsfreiheiten von Unternehmern langfristig erheblich einschränken können.

Das Konzept der „Transkulturalität als Praxis“ hat sich in der Analyse als tauglich erwiesen, den Zusammenhang von Kultur und ökonomischem Handeln für die empirische Forschungspraxis zugänglich zu machen. Dabei bestätigte sich die Ausgangsüberlegung, dass Grenzen nichts naturhaft Gegebenes sind, sondern diskursiv hergestellte Konstrukte, die durch die Bezeichnungs- und Handlungspraxis (re-)produziert werden. Damit ist aber auch Zugehörigkeit keine Größe, die im Subjekt verkörpert wäre, sondern die durch die soziale und diskursive Praxis hergestellt wird - und damit prinzipiell auch veränderlich ist. Dieser Aspekt wird bei wirtschaftsgeographischen Arbeiten, die auf die „kulturelle Embeddedness“ verweisen und dies an vermeintlich „geteilten Normen und Werten“ eines Kollektivs festmachen, häufig übergangen.

Dadurch, dass Grenzziehungen das Innen vom Außen trennen und damit Gemeinschaften herstellen, sind sie immer auch ein Akt der Identitätskonstruktion. Denn über symbolische Markierungen wird Differenz hergestellt, und das Ich setzt sich zum Anderen in Beziehung. Solche (Selbst-)Verortungsprozesse müssen als wesentliches Element beachtet werden, wenn kulturelle Aspekte unterneh- 
merischen Handelns untersucht werden sollen. So offenbarten die Analysen der Unternehmerbiographien, dass sich Unternehmer wie alle handelnden Subjekte - in einem Feld bewegen, in dem permanent Identitätspositionen verhandelt werden. In unternehmensrelevanten Interaktionen werden laufend Identitäten konstruiert, angenommen und abgelehnt. Die Vorstellung von einer „wesenhaften“ Identität sollte dabei aber ebenso aufgegeben werden wie die Vorstellung einer „wesenhaften“ Zugehörigkeit zu einer Gemeinschaft. Wie die Biographien vielmehr bestätigten, ist der Begriff der „Verortung" adäquater als der Begriff der "Identität“, da in ihm mit dem Handlungsakt (sich verorten), dem Zuschreibungseffekt (verortet werden) und dem situativen Charakter (Verortung als immer wieder neu kontextualisiertes Phänomen) drei Aspekte zusammenfließen, die für die Konstruktion von Identitätspositionen als konstitutiv identifiziert werden konnten.

Der Aspekt der Verortung wird als zentral angesehen, um in vermeintlich rein „ökonomischen "Interaktionssituationen die Handlungen der Akteure verstehen zu können. Aus diesem Gesichtspunkt lässt sich auch ableiten, dass „Transkulturalität als Praxis“ nicht nur als Analysekonzept gelesen werden kann, sondern gleichermaßen die Handlungspraxis konkreter Subjekte bezeichnet. Diese können sich nämlich in mehreren imaginären Gemeinschaften verorten, an deren Konstruktion sie permanent beteiligt sind. Sie besitzen damit prinzipiell die Fähigkeit, kontextbezogen auf unterschiedliche symbolische Deutungsschemata zu rekurrieren und im Handeln einzusetzen. Eine grundsätzliche Kompetenz zu solchermaßen „alltäglicher Transkulturalität" kann dabei jedem Unternehmer zugesprochen werden, weil in jeder ökonomischen Interaktion (meist unausgesprochen) „verhandelt“ wird, welche Deutungsschemata die Situation definieren.

Wie die Arbeit gezeigt hat, kann die Fähigkeit zur Transkulturalität auf der Ebene des einzelnen Akteurs weiter ausdifferenziert werden.

- „Alltägliche Transkulturalität" bezeichnet Handlungsroutinen, in deren Rahmen Akteure situativ Deutungsschemata heranziehen, um z.B. in ökonomischen Interaktionssituationen Bedeutungsgleichheit mit ihren Interaktionspartnern herzustellen. Sol- 
che Routinen sind - in terminologischer Anlehnung an Giddens (1997: 57) - im „praktischen Bewusstsein“ verankert. Sie stehen dem Handelnden in einer reflexiven Auseinandersetzung damit i.d.R. nicht zur Verfügung. ${ }^{28}$

- „Strategische Transkulturalität“ bezeichnet dagegen ein absichtsvolles Handeln, das mit einer reflexiven Verortung in unterschiedlichen Bezugssystemen einhergeht. In wiederkehrenden Interaktionssituationen verhandelte Deutungsschemata können dem Akteur im „diskursiven Bewusstsein“ zur Verfügung stehen. Das versetzt ihn in die Lage, mit Identitätskodierungen flexibel umzugehen und sich situationsabhängig und intentional auf unterschiedliche Bezugssysteme einzustellen. Strategische Transkulturalität, das Beispiel von Kevsan (vgl. Fall 5) hat es sehr deutlich herausgestellt, ist damit eine ökonomisch verwertbare Ressource, die Handlungsspielräume bei der Marktbearbeitung erweitert und dem Unternehmer die Teilhabe an unterschiedlichen Netzwerken ermöglicht.

Prinzipiell ist die Ausprägung der Fähigkeit zu alltäglicher oder strategischer Transkulturalität von der Heterogenität der Kontexte abhängig, in denen ein Unternehmer agiert bzw. mit denen er im Laufe seiner Biographie konfrontiert worden ist. Die Inwertsetzung von Transkulturalität als Ressource setzt einen individuellen Lernprozess voraus, weswegen der Zugang über die Biographie als eine geeignete Methode betrachtet werden kann, um die Kompetenz zur flexiblen Selbstverortung nachzuvollziehen.

Zusammenfassend lässt sich feststellen, dass das Konzept „Transkulturalität als Praxis“ hilft, die kulturellen Aspekte ökonomischen Handelns zu erschließen, indem es den Blick auf die Praxis der Herstellung und Wirkung kultureller Grenzziehungen richtet. Gleichzeitig werden kulturelle Grenzen nicht mehr als „zwischen“ Subjek-

28 Das ist auch ein Grund dafür, dass die in dieser Arbeit verwendeten Methoden bei der Analyse „alltäglicher Transkulturalität“ an ihre Grenzen stießen. Unreflektierte Handlungsroutinen sind in Interviews kaum erschließbar. 
ten, sondern als „innerhalb“ handelnder Subjekte liegend konzeptualisiert. Hierdurch werden empirisch feststellbare Mehrfachzugehörigkeiten von Akteuren auch aus theoretischen Überlegungen akzeptiert und der kontextabhängige, reflexive Umgang mit Deutungsschemata einer Analyse zugänglich gemacht.

Der flexible Umgang mit Identitätskodierungen und die prinzipielle Überschreitbarkeit von Grenzen führen jedoch nicht zu deren Auflösung. Im Gegenteil: Der Rückgriff auf einen Diskursstrang in einer konkreten Interaktionssituation, unabhängig davon ob unbewusst/alltäglich oder bewusst/strategisch, geht mit einer Reproduktion und damit einer Stabilisierung des entsprechenden Diskurses einher. Gleichzeitig erschließt „Transkulturalität als Praxis“ konzeptionell aber auch kulturellen Wandel. Denn dadurch, dass es den "Aushandlungsprozess" von Deutungsschemata in Interaktionen in den Vordergrund stellt, ist auch potenzielle Veränderung und damit der prozessuale Charakter von Kultur mitgedacht.

Kulturelle Grenzziehungen, d.h. die Herstellung von Innen-Außen-Differenzen entlang von Symbolen, sind nicht nur "national“ etikettiert oder an Herkunft gebunden. Zugehörigkeit zu einer bestimmten imaginären Gemeinschaft wird an sehr unterschiedlichen und vielfältigen Symbolen markiert und kommuniziert - in der Arbeit wurde auf einige hingewiesen. Das Konzept der „Transkulturalität als Praxis" kann daher als offen für eine Übertragbarkeit auf andere Forschungsfragen angesehen werden, bei denen es um Zusammenhänge von „Kultur und Handeln“ geht. 